\title{
Attention to Hierarchical Level Influences Attentional Selection of Spatial Scale
}

\author{
Anastasia V. Flevaris, \\ Department of Psychology, University of California at Berkeley, and Veterans Administration \\ Center, Martinez, CA \\ Shlomo Bentin, and \\ Department of Psychology and the Center of Neural Computation, Hebrew University of \\ Jerusalem \\ Lynn C. Robertson \\ Department of Psychology, University of California at Berkeley, and Veterans Administration \\ Center, Martinez, CA
}

\begin{abstract}
Ample evidence suggests that global perception may involve low spatial frequency (LSF) processing and that local perception may involve high spatial frequency (HSF) processing (Shulman, Sullivan, Gish, \& Sakoda, 1986; Shulman \& Wilson, 1987; Robertson, 1996). It is debated whether SF selection is a low-level mechanism associating global and local information with absolute LSF and HSF content, or whether it is a higher level mechanism involving a selective process that defines the SF range in which global and local can then be relatively defined. The present study provides support for the latter claim by demonstrating that allocating attention to global or local levels of hierarchical displays biased selection of LSFs or HSFs, respectively, in subsequently presented compound gratings. This bias occurred despite a change in the response dimension (from letter identification in the hierarchical stimulus to orientation discrimination in the grating) and despite a difference in retinal location of the hierarchical stimuli and the grating stimulus. Moreover, the bias was determined by the relationship between the $2 \mathrm{SFs}$ in the compound grating (i.e., their relative frequency) rather than the absolute SF values.
\end{abstract}

\section{Keywords}

hierarchical perception; selective attention; spatial frequency

\begin{abstract}
Studies of processing hierarchically structured visual stimuli have found separate neural mechanisms biased toward global versus local processing (e.g. Heinze, Hinrichs, Scholz, Burchert, \& Mangun, 1998; Heinze \& Munte, 1993; Ostwald, Lam, Li, \& Kourtzi, 2008), but the perceptual mechanisms involved continue to be debated. Much of the work in this domain has employed hierarchical letter displays (aka "Navon" patterns). In these displays, the spatial arrangement of a set of (local) letters forms a larger (global) letter, and participants report targets at either the global or the local level. Although there has been general agreement that visual areas in each hemisphere function differently in processing these levels, the specific level of processing and the putatively related neural mechanism
\end{abstract}


where the difference occurs have varied across neuropsychological (Delis, Robertson, \& Efron, 1986; Lamb, Robertson, \& Knight, 1989; Robertson \& Delis, 1986; Robertson \& Lamb, 1991; Robertson, Lamb, \& Knight, 1988), electrophysiological (Han \& Chen, 1996; Han, Liu, Yund, \& Woods, 2000; Heinze et al., 1998), and functional imaging (Fink et al., 1997; Han et al., 2002; Martinez et al., 1997; Weissman \& Woldorff, 2005) studies.

Whatever the precise neural mechanism might be, in concert, these previous studies provide evidence that allocating attention to and processing of targets at the global level of a hierarchical display elicits a different pattern of brain activity than allocating attention to and processing of targets at the local level. These differences occur even though the same stimulus set is presented and the same response type is initiated, with only instructions to attend to the global or local level being varied.

The distinction between global and local levels is obvious in a Navon pattern, but this distinction becomes more complicated when considering the visual stimuli outside the laboratory. Our environment is filled with objects that are hierarchically structured (e.g., trees making up a forest; books making up a bookshelf; eyes, nose, and mouth making up a face), but a local level in one instance (e.g., local trees in a global forest) could be a global level in another instance (e.g., local leaves in a global tree), depending on perceptual intentions that direct the focus of attention. To explain this flexibility, theories of processing hierarchical displays must provide an account for how the global and local levels are initially selected. One possibility is that the visual system defines global and local information through low-level perceptual channels that respond to different spatial frequency (SF) content. In fact, Sergent (1982) suggested that the hemispheric differences noted above reflect a slightly different bias toward lower or higher SF channels in the right and left hemispheres, respectively. Another account is based on the idea that attentional selection of global or local levels involves a process that interacts with early visual channels that are invariant to overall spatial scale. This possibility formed the basis of an alternative model to Sergent's, a model in which a secondary perceptual process guides selection of relatively lower or higher SFs (Ivry \& Robertson, 1998; Robertson \& Ivry, 2000). The present set of studies addresses the issue of tuning to absolute versus relative SFs that distinguishes these two models.

It has been well established that responding to single sinusoidal SF gratings affects responses to the global or local level in Navon patterns (e.g., Shulman, Sullivan, Gish, \& Sakoda, 1986; Shulman \& Wilson, 1987). For instance, gratings of a particular SF were shown to participants for several minutes in order to fatigue (adapt) the neural response in that SF channel (see De Valois \& De Valois, 1990) and were followed by a Navon pattern. Participants were slower to report targets at the global level after low spatial frequency (LSF) than high spatial frequency (HSF) adaptation and slower to report targets at the local level after HSF than LSF adaptation. Similarly, the detection threshold for frequencies following a global response was lower for LSF gratings and lower for HSF gratings following a local response (Shulman \& Wilson, 1987).

The relationship between SF selection and processing targets in hierarchical displays was further studied by Robertson (1996) with Navon patterns that varied in their SF content. In that study, participants discriminated letter targets that could appear at either the global or local level of Navon patterns; they were faster at reporting a target in trial $n$ when it appeared at the same level as in trial $n-1$ (i.e., same-level priming). It is important to note that same-level priming occurred whether or not the color, shape, contrast, polarity, or location of the displays changed from trial to trial, but it was eliminated when the SF content of the displays changed between trials. In alternating trials, the Navon patterns were contrast-balanced to filter out the lower SFs. This was done by adding a set of darkened dots to each pixel of the display such that the mean luminance across each set of dots (including 
the central brightened pixel) summed to the mean luminance of the background. An important finding was that when the trials alternated between contrast-balanced and fullspectrum displays, same-level priming disappeared. That is, SF was the only dimension that required consistency across trials for the same-level priming to occur. Robertson concluded that SFs are used to guide attention to one or the other level of hierarchical displays. In a later study, Lamb, Yund, and Pond (1999) replicated this finding, but found that if participants had previous experience in a block of contrast-balanced displays, same-level priming was then observed in an alternating (between contrast-balanced and full-spectrum) condition. Given these findings, Lamb et al. argued that hierarchical displays are not necessarily parsed on the basis of their SFs as Robertson (1996) had proposed. However, as Robertson (1999) pointed out in a response article, although these results may constrain the attention-guiding hypothesis, they do not entirely explain her earlier findings. It is clear that SFs are involved in parsing information into global and local levels when SF differences between the levels are present, although other features of a stimulus can also be valuable in parsing hierarchical levels under conditions when SF differences are not present.

Although the extent to which SF is used to guide attention in hierarchical displays is disputed, it is clear that processing such displays is at least partially flexible, and perceptual strategies can be applied to basic visual properties of the image depending on the demand characteristics of the task. In the three experiments reported here, we further explored the nature of this relationship and asked whether selection of higher or lower frequencies in a compound grating would be influenced by previously attending to the global or local level of a Navon pattern. In all three experiments, participants viewed pairs of Navon patterns as the "prime" display (one in each visual field) and were asked to make same-different judgments on either the global or the local level. Following the response to the Navon patterns on each trial, one or two compound gratings with two orthogonally oriented SFs appeared as the "probe" display, and participants were instructed to make an orientation judgment about either the "thick lines" (LSFs) or the "thin lines" (HSFs). The global-local and the LSF-HSF conditions were orthogonally associated, forming four possible combinations that were presented in a counterbalanced block design. In Experiment 1, two identical compound gratings were presented in the periphery at the same locations as the two Navon patterns. In Experiment 2, only one compound grating was presented after the two peripheral Navon patterns, and it was centrally located. In Experiment 3, we directly examined whether attention to hierarchical level biases attention to relative or absolute SF by presenting a 1.8 cycle/degree grating as the relatively higher SF in the compound grating during half of the blocks and as the relatively lower SF in the compound grating during the other half. In all three experiments, the two SFs within the compound grating differed in orientation, forcing participants to selectively attend to the task-relevant SF in order to report its orientation. Thus, in contrast to the previous studies (e.g., Shulman \& Wilson, 1987; Robertson, 1996), the priming effects examined in the present experiments concerned the influence of hierarchical perception on selective attention to SF rather than the perceptual processing of low versus high SFs per se. If selection of SFs is, indeed, influenced by attending to global versus local levels, participants should be faster at selectively reporting the orientation of relatively LSF than relatively HSF gratings during the global attention block, and they should be faster at selectively reporting the orientation of the relatively HSF than relatively LSF gratings during the local attention block.

\section{Experiment 1}

\section{Method}

Participants-Sixteen students from the University of California, Berkeley (eight women) participated in the experiment for course credit. All had normal or corrected-to-normal 
vision. The experiment was approved by the University of California, Berkeley Internal Review Board, and all participants gave informed consent before participating.

Stimuli-The Navon patterns were generated using Adobe Photoshop. Seen from a distance of $1 \mathrm{~m}$, each local letter subtended $0.7^{\circ}$ of visual angle and was black Helvetica bold font presented on a gray background, arranged to form a global letter that was $4.0^{\circ}$ wide by $5.7^{\circ}$ high. The letters used were $A, C, D, E, F$, and $H$, in all their global and local combinations, with the exception of congruent combinations (e.g., a global $A$ made up of local $A$ s). This resulted in 30 different Navon displays.

The compound gratings were generated in Matlab (Mathworks, Natick, MA) with a sinusoid function for each SF. Each compound grating subtended $5.4^{\circ}$ of visual angle and was composed of a 0.7 cycle/degree ( 4 cycles/image) grating (the relatively LSF component) and a 2.4 cycle/degree (13 cycles/image) grating (the relatively HSF component). Both SF gratings were at $100 \%$ contrast. The relatively LSF component was oriented either at $45^{\circ}$ (tilted to the right) or at $135^{\circ}$ (tilted to the left) and the relatively HSF component was oriented in the opposite direction (see Figure 1).

Procedure-The stimuli were shown on a Dell 17-in. color monitor with a vertical refresh rate of $60 \mathrm{~Hz}$ at a resolution of $1,024 \times 768$ pixels. Participants were seated $1 \mathrm{~m}$ from the screen in a dimly lit room. Trial timing was controlled by Presentation (Neurobehavioral Systems, Albany, CA) and is depicted in Figure 1. Each trial began with a central fixation cross presented for $500 \mathrm{~ms}$, followed by two Navon patterns (the prime), one in the left and one in the right visual field, with the medial edge $1^{\circ}$ from fixation. The letters remained on the screen until response. Participants indicated whether the two Navon displays were the same or different at the global level during half of the trials, and they indicated whether the Navon displays were the same or different at the local level during the other half of trials. Responses were made by pressing one of two keys on the keyboard (either $S$ for same or $D$ for different, or $J$ for same or $K$ for different) with either the left or right hand, which was also counterbalanced across participants. That is, half of the participants used the $S$ and $D$ keys (left hand), and the other half used the $J$ and $K$ keys (right hand) for the prime task. Immediately following the Navon prime, two identical compound gratings (the probe) appeared until response in the same spatial locations as the Navon patterns.

Participants indicated whether the relatively LSF components (referred to as "thick bars") were oriented to the left or right in half of the trials, and they indicated whether the relatively HSF components (referred to as "thin bars") were pointing to the left or right in the other half of the trials. Responses to the grating probe were made by pressing one of two keys on the keyboard (the opposite pair of keys than the pair used for the prime task) by the opposite hand than that used for the prime task. Participants thus performed four blocks of 120 trials each, the order of which was counterbalanced. A different combination of prime and probe tasks was used in each block. In one block, participants responded to the global level and then indicated whether the LSF was oriented left or right. In another block, they responded to the global level and then indicated whether the HSF was oriented left or right. In another block of trials, they responded to the local level and then indicated whether the LSF was oriented left or right. Finally, in another block, they responded to the local level and then indicated whether the HSF was oriented left or right. Prior to each experimental block, participants were given 30 practice trials to get them used to the task. Each session lasted about $30 \mathrm{~min}$. 


\section{Results}

Probe reaction times (RTs)_Across priming conditions, probe-performance accuracy was at ceiling (97\%) and was not analyzed further. RTs to the designated SF in the probe were analyzed with a $2 \times 2$ analysis of variance (ANOVA) with repeated measures. The factors were primed level (global vs. local) and attended SF (LSF vs. HSF). RTs to incorrect responses, either to the prime or to the probe, and those above or below 2 standard deviations of the mean (calculated within-subject and within-condition) were excluded ( $16 \%$ of total trials; $11 \%$ for incorrect responses and $5 \%$ for outlier RTs). ${ }^{1}$ This analysis revealed a significant interaction between the primed level and attended SF, $F(1,15)=7.1$, $M S E=1293.3 p<.05$, partial $\eta^{2}=.32$, but no significant main effects of SF or primed level. That is, whereas the overall means were statistically equivalent across the two SF conditions ( $530 \mathrm{~ms}$ to LSF and $534 \mathrm{~ms}$ to HSF) and across the two primed levels (534 ms in the global attention blocks and $531 \mathrm{~ms}$ in the local attention blocks), mean RTs were faster to LSF probes following global $(519 \mathrm{~ms}, S E=22 \mathrm{~ms})$ than local $(540 \mathrm{~ms}, S E=23 \mathrm{~ms})$ primes, and faster to HSF probes following local ( $520 \mathrm{~ms}, S E=26 \mathrm{~ms}$ ) than global ( $548 \mathrm{~ms}, S E=35 \mathrm{~ms}$ ) primes (see Figure 2). Whereas some subjects showed both simple effects (faster RTs to LSF targets in the global block and to HSF probes in the local block), other subjects demonstrated only one of the simple effects (e.g., faster RTs to LSF probes in the global block but no difference in the local block or vice versa). Nonetheless, the significant interaction shows an overall pattern of better performance for LSF than HSF probes when attending to the global level and for HSF than LSF probes when attending to the local level. In Figure 3, the difference between RTs to LSF and HSF probes is plotted for each individual. All but two participants (shown in gray) had an increased mean difference in the local than global attention blocks, reflecting the consistency of the overall pattern of priming effects.

Prime RTs-We also analyzed RTs to the primes with a $2 \times 2$ ANOVA with repeated measures. The factors were attended hierarchical level (global vs. local) and whether the attended probe frequency was low or high. This analysis revealed a main effect of prime, $F(1,15)=7.3, M S E=9719.5, p<.05$, partial $\eta^{2}=.33$, indicating that participants were faster when responding to global $(683 \mathrm{~ms})$ than local $(746 \mathrm{~ms})$ primes. Although the direction of this effect is consistent with global precedence (Navon, 1977), there was evidence of a speed-accuracy trade-off in the prime responses: Accuracy was greater for local than global responses ( $94.6 \%$ and $89.9 \%$, respectively), as revealed in a main effect of attended level, $F(1,15)=101.6, M S E=3.8, p<.0001$, partial $\eta^{2}=.87$. It is important to note that this difference in prime accuracy did not interact with the (forthcoming) probe's frequency, $F(1,15)<1$. When we combined RT and accuracy into a single measure by dividing RT by proportion correct (Townsend \& Ashby, 1983) and reran the analyses using this measure, we found no effects in the prime data $\left(F_{\mathrm{s}}<1\right)$, and thus, no global precedence.

\section{Discussion}

The results of Experiment 1 demonstrate that attention to a given hierarchical level of a Navon pattern influences the time it takes to selectively respond to the relatively LSF or HSF in a subsequent compound grating. RTs to the relatively LSF were facilitated following attention to the global level of the Navon patterns, and response times to the relatively HSF in the same compound gratings were facilitated following attention to the local level. The current results extend the previous findings of Shulman and colleagues (1986; Shulman \& Wilson, 1987) because our study required participants to select a SF within a stimulus

\footnotetext{
${ }^{1}$ The same analysis performed without excluding trials showed a similar pattern, albeit noisier. The mean RTs were $522 \mathrm{~ms}$ and 542 $\mathrm{ms}$ following global and local processing, respectively, in the LSF blocks; and $547 \mathrm{~ms}$ and $536 \mathrm{~ms}$ following global and local processing, respectively, in the HSF blocks.
} 
compound containing both relatively low and relatively high SFs, whereas in their studies, participants were presented with simple gratings (either LSF or HSF). Moreover, the two SFs in our compound grating are not considered LSF and HSF in the absolute sense. Rather, our 2.4 cycle/degree HSF grating is a lower or mid-level SF in the spectrum (De Valois \& De Valois, 1988). Indeed, Shulman and colleagues (1986; Shulman \& Wilson, 1987) found that a 2 cycles/degree grating was associated with global perception, whereas higher SF gratings of 8 cycles/degree and 16 cycles/degree were associated with local perception. This is an important distinction because it is consistent with models claiming that the relationship between SF and attention to hierarchical level is more than an automatic early perceptual effect. A bottom-up priming effect would occur through stimulation of the same SF channels as biased by the prime rather than being contingent on the need to parse and then select relatively higher or lower SFs in the probe.

Before considering the implications of these findings, it is necessary to rule out a simple explanation of the results based on a prime-induced bias of the distribution of attention in space. Specifically, it is possible that the span of attentional focus varied between global and local blocks. Participants may have adopted a wide and more distributed attentional distribution when responding to the global level and a narrow, more focused attentional distribution when responding to the local level. Although we attempted to mitigate this problem by presenting hierarchical patterns in the periphery and asking participants to make same- different judgments at either the global or local level across the visual field, there was no need to change the spatial span of attention established by the prime in order to respond to the grating probes because the grating probes were presented in the same spatial locations as the primes. Furthermore, it is also possible that stimulus size was the critical dimension, rather than attention to hierarchical level. For example, if participants adopted a wider or narrower attentional window to perform the global versus local task, respectively, a larger attentional window could prime the larger LSFs, whereas a smaller attentional window could prime the smaller HSFs. Finally, we did not monitor eye movements in Experiment 1 , and thus we could not rule out the possibility that participants made more eye movements in one condition than another. These alternative hypotheses were tested and ruled out in Experiments 2A and 2B.

\section{Experiment 2}

In Experiment 2A, a single compound grating was presented as the probe at fixation after the same- different response to the pair of peripherally presented Navon patterns, and we monitored eye movements to verify that participants maintained central fixation during the prime task. In so doing, we assured that the spatial window required to perform the local prime task was actually closer in size to the window required for the LSF grating task than the window required to perform the global prime task. Because the prime task required a comparison of the two peripheral Navon patterns, the minimum spatial window required for the local attention block would need to span the nearest two local letters in the two displays. In Experiment $2 \mathrm{~A}$, each local letter subtended $0.7^{\circ} \times 0.7^{\circ}$ of visual angle, and their edges were located $1^{\circ}$ to the left and right of fixation, resulting in a spatial window with a $3.4^{\circ}$ diameter for the local prime task. For the global attention block, the spatial window would need to span nearly the whole $5.7^{\circ} \times 4^{\circ}$ global letters, resulting in a spatial window with a diameter somewhere between $11.4^{\circ} \times 6^{\circ}$. It is important to note that the compound grating probe had a diameter of $5.4^{\circ}$ centered at fixation, making it closer in size to the spatial window required for the local than global block. Thus, in a local block, participants would not have to alter the span of their spatial window to perform either the LSF or HSF grating orientation task because the central grating was presented within the span of spatial attention. If local primes facilitate performance on HSF rather than LSF grating probes, a pure spatial account of the results is unlikely. In addition to presenting a single compound 
grating probe at fixation and monitoring eye movements during both prime and probe, the Navon patterns in Experiment 2A were presented for $300 \mathrm{~ms}$ rather than until response, as in Experiment 1, to facilitate the maintenance of central fixation. It is important to note that the compound grating included the same SFs as in Experiment 1 as measured in the probe, but the change in its location from the peripheral to central visual field changed the spectral sensitivities of the visual response. In this way, the relative frequencies in the probe remained the same, whereas the input visual frequencies were vastly different.

Experiment $2 \mathrm{~B}$ was identical to $2 \mathrm{~A}$ in all respects except that the primes in Experiment $2 \mathrm{~B}$ were single letters that were either the same size as the global letters in Experiment 2A or the same size as the local letters. The letters were either the same or different and, as in Experiment 1, the response was to report whether they were the same or different letter as rapidly as possible. If size rather than SF was the critical factor in Experiment 1, then we would expect large letters to prime LSFs and small letters to prime HSFs. However, if attention to hierarchical level is critical, then we would expect no priming effects of size on SF.

\section{Method}

Participants-Sixteen students from the University of California, Berkeley (eight women) participated in Experiment 2A, and a different sample of 16 students from the same population (12 women) participated in Experiment 2B. They received course credit for their participation. All had normal or corrected-to-normal vision. The experiment was approved by the University of California, Berkeley Institutional Review Board, and all participants gave informed consent before participating.

Stimuli-The stimuli in Experiment 2A were identical to those used in Experiment 1 except that only one grating probe was presented. The grating subtended $5.4^{\circ}$ in diameter and appeared at central fixation.

The prime stimuli in Experiment 2B were single letters in black Helvetica bold font presented on a gray background. The small letters subtended $0.7^{\circ}$ of visual angle and were identical to those that appeared at the local level in Experiments 1 and 2A. The large letters were the same size as the global letters in Experiments 1 and $2 \mathrm{~A}, 4.0^{\circ} \times 5.7^{\circ}$ high. The grating probe in Experiment $2 \mathrm{~B}$ was identical to that used in Experiment $2 \mathrm{~A}$.

Procedure-The exact same procedure was used for Experiments $2 \mathrm{~A}$ and $2 \mathrm{~B}$, the only difference being whether the Navon patterns (2A) or single letters the size of the global letters or the size of the local letters (2B) appeared as the primes. The procedure was similar to Experiment 1 with three differences. First, the Navon/letter pairs were presented for 300 $\mathrm{ms}$, followed by the reappearance of the fixation cross until response. Second, eye movements were monitored to verify that the participants maintained fixation while the primes were present. Third, only one compound grating probe followed the primes, and it was centered at fixation. The grating probe remained on the screen until response. Other than those three differences, the procedure was identical to Experiment 1. Participants performed the same four blocks of 120 trials each, in counterbalanced order, and performed the same two tasks with the same counterbalanced response mappings.

\section{Results}

\section{Experiment 2A}

Probe RTs: Similar to Experiment 1, probe-performance accuracy was at ceiling (97\%) and therefore not analyzed further. RTs to the grating probes were analyzed with a $2 \times 2$ ANOVA with repeated measures; the factors were primed level (global vs. local) and 
attended SF (low vs. high). RTs to incorrect responses, either to the prime or to the probe, and those above or below 2 standard deviations of the mean were excluded, as well as any trials in which participants moved their eyes prior to the presentation of the grating probe ( $\sim 16 \%$ of total trials; $10 \%$ for incorrect responses and $6 \%$ for outlier RTs). ${ }^{2}$ Participants had no trouble maintaining fixation, and eye movements accounted for almost none of the excluded trials (i.e., the highest number of eliminated trials due to eye movements for a single participant was two). As found in Experiment 1, there was a significant interaction between primed level and SF, $F(1,15)=5.5, M S E=1380.9, p<.05$, partial $\eta^{2}=.27$, and there were no significant main effects. That is, whereas RTs did not significantly differ across the two SF conditions (551 ms for LSF probes and $536 \mathrm{~ms}$ for HSF probes) or across the two prime conditions (543 ms for both global and local primes), participants were faster reporting LSF orientation following global $(540 \mathrm{~ms}, S E=19 \mathrm{~ms})$ than local $(562 \mathrm{~ms}, S E=$ $19 \mathrm{~ms}$ ) primes, and they were faster reporting HSF orientation following local $(525 \mathrm{~ms}, S E=$ $27 \mathrm{~ms}$ ) than global $(546 \mathrm{~ms}, S E=30 \mathrm{~ms}$ ) primes (see Figure 4). Figure 5 presents the difference between mean RTs to LSF and HSF targets for each subject in Experiment 2A, showing a similar pattern to Experiment 1, although it was slightly more variable.

Prime RTs: We also analyzed mean RTs to the Navon primes with a $2 \times 2$ ANOVA with repeated measures with attended level (global vs. local) and attended SF in the probe (LSF vs. HSF target) as factors. This analysis revealed a main effect of attended level, $F(1,15)=$ 8.0, MSE $=13695.6, p<.05$, partial $\eta^{2}=.35$, indicating that participants were faster responding to global $(755 \mathrm{~ms})$ than local $(849 \mathrm{~ms})$ primes, as in Experiment 1. Again, there was no effect of the probe's SF and no interaction between attended level and SF of the probe, $F(1,15)<1$.

The analysis of prime accuracy revealed that, similar to Experiment 1, the main effect of attended level was qualified by a speed-accuracy trade-off. Although accuracy was high at both processing levels, the 2 (attended level) $\times 2(\mathrm{SF})$ repeated measures ANOVA for prime accuracies resulted in a main effect of prime level, $F(1,15)=98.9, M S E=15.1, p<.0001$, partial $\eta^{2}=.87$, with the responses to local $(95 \%)$ more accurate than to global $(85 \%)$ primes. As in Experiment 1, we also ran the prime analyses using the combined measure (RT/proportion correct) and found no effects of primes using this measure. However, despite the speed-accuracy trade-off found for the main effect of attended level, there was also a marginal interaction between attended level and SF, $F(1,15)=4.5, M S E=12.7, p=.051$, partial $\eta^{2}=.23$, which had the same pattern as the RTs. That is, accuracy to global primes was higher in the LSF probe condition (86\%) than HSF (84\%) probe condition, and accuracy to local primes was higher in the HSF (96\%) than LSF (93\%) probe conditions (see Table 1).

Comparison of experiments 1 and 2A: To explore whether the change in probe location from peripheral to central vision affected performance, we conducted a statistical comparison of Experiments 1 and 2 by running a mixed model ANOVA with experiment (1 vs. 2A) as a between-participants factor and prime level (global vs. local) and SF (LSF vs. HSF target) as within-participant factors. For the probe RTs, this analysis revealed no interactions with experiment. The only significant effect was the interaction between prime level and SF, $F(1,30)=12.5, M S E=1337.1, p<.001$, partial $\eta^{2}=.29$, reinforcing the outcome of the individual experiments.

\footnotetext{
${ }^{2}$ The same analysis performed without excluding trials showed a similar pattern. The mean RTs were $535 \mathrm{~ms}$ and $560 \mathrm{~ms}$ following global and local processing, respectively, in the LSF blocks; and $546 \mathrm{~ms}$ and $529 \mathrm{~ms}$ following global and local processing, respectively, in the HSF blocks.
} 
The same analysis conducted on the prime RTs and on the prime accuracies also mirrored the results found in the individual experiment analyses and did not reveal any interactions with experiment. For the prime RTs, there was a main effect of attended level, $F(1,30)=15$. $2, M S E=11207.5, p<.001$, partial $\eta^{2}=.34$, reflecting the fact that RTs to global primes were faster $(724 \mathrm{~ms})$ than RTs to local primes $(797 \mathrm{~ms})$. For the prime accuracies, as was found in both experiments, there was a main effect of attended level, $F(1,30)=164.5, M S E$ $=19.1, p<.001$, partial $\eta^{2}=.85$, indicating that participants were more accurate in the local (94.6\%) than global (84.6\%) task (which, in concert with the RT results, might indicate a speed-accuracy trade-off). There was also an interaction between prime and the attended SF in the probe, $F(1,30)=5.1, M S E=10.3, p<.01$, partial $\eta^{2}=.15$. Follow-up $t$ tests revealed a trend for responses to global primes to be more accurate in the LSF than HSF block, $t(31)$ $=1.871, p=.07, d=1.9$, whereas there was no such difference for responses to local primes. In sum, the statistical comparison of Experiments 1 and 2A mirrored the results of the individual experiments' analyses and, most important, did not show any interaction with experiment.

Experiment 2B-Accuracy rates to both the grating probes and to the letter primes in Experiment 2B were at ceiling ( $96 \%$ and $95 \%$, respectively) and were therefore not analyzed further. RTs to the grating probes and to the letter primes were analyzed with a $2 \times 2$

ANOVA with repeated measures; the factors were primed size (large vs. small) and attended SF (low vs. high). RTs to incorrect responses, either to the prime or to the probe, and those above or below 2 standard deviations of the mean were excluded, as well as any trials in which participants moved their eyes prior to the presentation of the grating probe ( $\sim \%$ of total trials). No significant main effects emerged. More important, there was not even a trend toward an interaction between size and $\mathrm{SF}(F \mathrm{~s}<1)$.

Comparison of experiments 2A and 2B: To compare Experiments 2A (Navon prime) and 2B (size prime), we conducted a mixed model ANOVA with experiment (A vs. B) as a between-participants factor, and prime level/size (global/large vs. local/ small) and SF (LSF vs. HSF target) as within-participant factors. For the probe RTs, this analysis revealed a three-way interaction between the factors SF, level/size, and experiment, $F(1,30)=4.1$, $M S E=11353.8, p=.05$, partial $\eta^{2}=.12$. This interaction reflects the fact that we found a significant interaction between primed level and attended SF in Experiment 2A (with Navon patterns as primes) and no such interaction between primed size and attended SF in Experiment 2B (with large or small letters as primes). No other effects were significant for the probe RTs. For the prime RTs, the same analysis revealed a main effect of size/ level, $F(1,30)=4.9, M S E=11714.2, p<.05$, partial $\eta^{2}=.14$, reflecting the fact that participants were faster at global/large primes $(548 \mathrm{~ms})$ than local/small primes $(553 \mathrm{~ms})$. However, as we found in the individual experiment analyses, this difference was only significant in Experiment $2 \mathrm{~A}$ for the global versus local primes (al though qualified by a speed-accuracy trade-off) and not in Experiment 2B for the large versus small primes. This was corroborated by a two-way interaction between size and experiment, $F(1,30)=4.5, M S E=11714.2, p<$. 05 , partial $\eta^{2}=.13$. There were no other significant effects in the prime RT analyses.

\section{Discussion}

The results of Experiment 2A replicated those of Experiment 1. Responses to LSFs in compound gratings were faster following attention to global than local levels in the primes, whereas responses to HSFs in the same gratings were faster following attention to local than global levels in the primes. Hence, the influence of attending to a particular hierarchical level on SF selectivity was found despite a change in spatial location of the probe from periphery to central vision and despite the fact that the location of the hierarchical primes and compound grating probes changed. These findings question a simple spatial focus 
account of the results, and by monitoring eye movements, we assured that the Navon patterns and the compound gratings appeared at different regions on the retina. Moreover, in Experiment $2 \mathrm{~B}$ when we presented single letters of identical size to the global and local letters in Experiment 2A, we did not find priming of size on SF. This is strong evidence that attention to hierarchical level was the critical factor in priming the selection of a SF, and not attention to size per se.

An important result was that the same pattern of priming was found in Experiments 1 and $2 \mathrm{~A}$ despite the fact that perceptual sensitivity to different SFs varies between the periphery and the fovea, with a better resolution of HSFs in the fovea than the peripheral visual field (De Valois \& De Valois, 1990). Moreover, it is noteworthy that both SFs that we used in the compound grating were at the lower end of the human visible spectrum and, without priming, both have been previously associated with global processing when presented alone (Shulman et al., 1986; Shulman \& Wilson, 1987). Hence, the complex grating stimulus in the current study required participants to selectively attend to the relatively lower or relatively higher of two objective LSF gratings.

Nevertheless, relations between the hierarchical patterns and SF might have been different here than in previous studies, so it is important to directly examine hierarchical attention and its relationship to relative SF and absolute SF. To this end, in Experiment 3 the same 1.8 cycles/degree grating was the relatively lower SF of the compound grating probe during half of the blocks and was the relatively higher SF of the compound grating probe during the other half of the blocks. If attention to hierarchical level triggers a process that biases attentional selection of relative SF rather than absolute SF, attention to global primes should facilitate attention to the 1.8 cycles/degree grating when it is relatively lower and attention to local primes should facilitate attention to the same 1.8 cycles/degree grating when it is relatively higher.

\section{Experiment 3}

\section{Method}

Participants-Sixteen students from the University of California, Berkeley (10 women) participated in the experiment for course credit. All had normal or corrected-to-normal vision. The experiment was approved by the University of California, Berkeley Institutional Review Board, and all participants gave informed consent before participating.

Stimuli-The Navon patterns used as primes were identical to those used in Experiments 1 and $2 \mathrm{~A}$. There were two compound grating probe conditions: LOW and HIGH, referring to the absolute SF differences between the two grating probes. The LOW compound gratings were composed of a 0.9 cycles/degree ( 3 cycles/image) grating (the relatively LSF component) and a 1.8 cycles/degree (6 cycles/image) grating (the relatively HSF component), and the HIGH compound gratings were composed of a 1.8 cycles/degree grating (the relatively LSF component) and a 5.3 cycles/degree (18 cycles/image) grating (the relatively HSF component). Seen from $1 \mathrm{~m}$, the grating probes in both conditions subtended $3.4^{\circ}$ of visual angle and were presented at fixation akin to Experiment 2. As in the previous two experiments, the relatively LSF component was oriented either at $45^{\circ}$ (tilted to the right) or at $135^{\circ}$ (tilted to the left) and the relatively HSF component was oriented in the opposite direction (see Figure 6).

Procedure-The procedure was identical to that of Experiment 2A, except that participants performed eight blocks of 60 trials. The same four blocks as the previous two experiments (global/ lower, global/higher, local/lower, local/higher) were performed once with the LOW grating probes and once with the HIGH grating probes. 


\section{Results}

Probe RTs-Similar to Experiments 1 and 2, probe-performance accuracy was at ceiling (96\%) and therefore not analyzed further. RTs to incorrect responses, either to the prime or to the probe, and those above or below 2 standard deviations of the mean were excluded ( 16\% of total trials; $9 \%$ for incorrect responses and 7\% for outlier RTs). ${ }^{3}$ Eye movements were monitored but did not account for any of the excluded trials because participants had no trouble maintaining fixation. RTs to the grating probes were analyzed with a $2 \times 2 \times 2$ ANOVA with repeated measures. The factors were primed level (global vs. local), absolute SF condition (LOW vs. HIGH), and relative SF attended in the probe (lower vs. higher) as factors. This analysis revealed a significant interaction between primed level and relative $\mathrm{SF}, F(1,15)=6.6, M S E=6999.2, p<.05$, partial $\eta^{2}=.30$, and no other significant effects. Particularly noteworthy is that there were no main effect of the absolute spatial frequency and no interactions with this factor. Participants were faster reporting the relatively LSF orientation following global ( $555 \mathrm{~ms}, S E=22 \mathrm{~ms})$ than local $(574 \mathrm{~ms}, S E=28 \mathrm{~ms})$ primes, and they were faster reporting relatively HSF orientation following local $(530 \mathrm{~ms}, S E=31$ $\mathrm{ms}$ ) than global (586 ms, $S E=51 \mathrm{~ms}$ ) primes (see Figure 7). In Figure 8, the probe RTs to relatively lower SF and relatively higher SF grating probes following global and local level primes are plotted separately for the absolute LOW condition and absolute HIGH condition. The second-order interaction was not significant, $F(1,15)=1.2$. As indicated by the lack of interaction with absolute SF, priming was contingent on relative SF despite the fact that the same 1.8 cycles/ degree grating was the relatively higher SF in the absolute LOW condition and the relatively lower SF in the absolute HIGH condition.

Prime RTs-We also analyzed mean RTs to the Navon patterns with a $2 \times 2 \times 2$ ANOVA with repeated measures with absolute SF (LOW vs. HIGH), relative SF (lower vs. higher), and attended level (global vs. local) as factors. This analysis revealed a main effect of attended level, $F(1,15)=10.3, M S E=10359.7, p<.01$, partial $\eta^{2}=.41$, indicating that, as in Experiments 1 and 2, participants were faster responding to global $(635 \mathrm{~ms})$ than local $(692 \mathrm{~ms})$ primes. There was also an interaction between relative SF and attended level, $F(1$, $15)=5.5, M S E=2833.8, p<.05$, partial $\eta^{2}=.27$. Participants were faster when attending to the global than local prime level in both relative SF conditions, but this difference was much greater in blocks when they were attending to the relatively lower SF (80-ms difference) than the relatively higher SF (36-ms difference), $t(15)=2.4, p<.05, d=0.54$. It is important that, like in the analysis of the probe RTs, there were no interactions with absolute SF and no other effects in the prime RT analyses.

The analysis of prime accuracy revealed that, similar to Experiments 1 and $2 \mathrm{~A}$, the main effect of attended level was qualified by a speed-accuracy trade-off. That is, the 2 (absolute $\mathrm{SF}) \times 2$ (relative $\mathrm{SF}) \times 2$ (attended level) repeated measures ANOVA for prime accuracies revealed a main effect of attended level, $F(1,15)=93.2, M S E=7.4, p<.0001$, partial $\eta^{2}=$. 86 , with more accurate responses to local (93\%) than to global (88\%) primes. There was also an interaction between relative $\mathrm{SF}$ and level, $F(1,15)=8.0, M S E=8.4, p<.05$, partial $\eta^{2}=.35$, showing a greater difference between global and local primes in blocks when participants were attending to relatively lower SFs (6\% difference) than higher SFs (3\% difference), $t(15)=2.8, p<.05, d=1.0$. Similar to Experiments 1 and $2 \mathrm{~A}$, the analysis of the primes in Experiment 3 using the combined measure (RT/proportion correct) revealed no statistically significant effects.

\footnotetext{
${ }^{3}$ The same analysis performed without excluding trials showed a similar pattern. In the absolute LOW blocks, the mean RTs were 569 $\mathrm{ms}$ and $579 \mathrm{~ms}$ following global and local processing, respectively, in the relative LSF blocks; and $565 \mathrm{~ms}$ and $514 \mathrm{~ms}$ following global and local processing, respectively, in the relatively HSF blocks. In the absolute HIGH blocks, the mean RTs were $528 \mathrm{~ms}$ and $572 \mathrm{~ms}$ following global and local processing, respectively, in the relative LSF blocks; and $567 \mathrm{~ms}$ and $548 \mathrm{~ms}$ following global and local processing, respectively, in the relatively HSF blocks.
} 


\section{Discussion}

The results of Experiment 3 replicated the pattern of level priming that was found in Experiments 1 and 2A, with RTs to relatively lower SFs in the probe faster following global than local attention, and RTs to relatively higher SFs in the probe faster following local than global attention. It is important to note that this interaction was found for relative rather than absolute SFs as shown by the fact that the same 1.8 cycles/degree grating was relatively higher in half of the blocks and relatively lower in the other half of the blocks. That there were no effects or interactions with absolute SF is strong evidence that the priming effect is not merely due to lower level attentional tuning to SF per se but rather to a process that keeps the relatively global and local levels constant over large changes in stimulus location and frequency content.

\section{General Discussion}

In three experiments, we examined how same-different judgments about the global or local levels of information in pairs of hierarchical displays can affect subsequent allocation of attention to relatively high and low SFs in a compound grating. Although a direct link between the attended level in a hierarchical display (global vs. local) and responses to simple sinusoidal gratings has long been known (Shulman et al., 1986; Shulman \& Wilson, 1987), the mechanism by which attention to a given level (global or local) affects the selection of SFs in an image has not been sufficiently characterized before. Using HSF and LSF compound gratings rather than simple sinusoidal gratings, the present data indicate that attending to the local level facilitates the selection of the relative HSFs in the image and attending to the global level facilitates the selection of the relative LSFs. We also showed that this pattern of facilitation could not be explained by attention to size alone; large letters did not prime LSFs, nor did small letters prime HSFs (Experiment 2B).

We found the interaction between attended level and SF selection both when the grating probes appeared at the same peripheral locations as the Navon patterns (Experiment 1) and when they appeared at fixation (Experiment 2A), inconsistent with a simple spatial focus account of the results, and arguing against a simple early visual account for the priming effect (for example, that given the different spatial resolution of the rods and the cones, images presented in the periphery would preferentially activate lower SF channels than images presented at fixation). In fact, previous studies suggested that the 3 cycles/degree grating shows optimal contrast sensitivity at the fovea, whereas lower SFs of 0.6 cycles/ degree to 1.2 cycles/degree show optimal contrast sensitivity at $1.7^{\circ}$ eccentricity (Henriksson, Nurminen, Hyvärinen, \& Vanni, 2008). Thus, in Experiment 1, the relatively LSFs in the compound grating (i.e., 0.7 cycles/degree at $1^{\circ}$ eccentricity) were perceptually optimal, whereas in Experiment 2, the relatively HSFs in the compound grating were perceptually optimal (i.e., 2.4 cycles/ degree at fixation). The fact that we found the same pattern of results despite these early level differences across Experiments 1 and 2 is further evidence that this is a top-down effect that influences selectivity to the relative spatial scale.

More direct evidence for a selective attention effect was found in Experiment 3, where the selection of relative SFs interacted with attended prime level, and no effect of absolute SFs emerged. These findings strongly suggest that global versus local attention involves a flexible mechanism by which attention selects relatively lower versus relatively higher SFs, respectively. If the priming effects were due solely to the visual response to spectral information, we would have found the same pattern of results for the 1.8 cycles/degree grating whether it was the relatively lower or relatively higher frequency in the probe. Instead, the priming effects did not interact with absolute SF but only with relative SF, providing strong support that a flexible, top-down mechanism is involved in parsing global versus local levels of hierarchical images. Whereas an effect of relative SF is incompatible 
with a pure bottom-up mechanism, a flexible mechanism is compatible with the selection of both absolute and relative SFs. We are not arguing that absolute SFs are irrelevant. They may well set the spatial limits of what is visually available at any instant in time, but the selection of SFs can be modulated by top-down influences that link relative hierarchical levels across time and space.

The current study also demonstrates that level of processing (global or local) priming in Navon stimuli can extend to a completely unrelated stimulus and task. Robertson (1996) suggested that when stimuli were similar in structure (multileveled), selecting a given level on one trial to make a certain type of response (identification) automatically primed the selection of that same level on the next trial to make a similar type of response, whether or not the two stimuli were in the same or different locations. The current results extend the previous ones showing that the selected level in a Navon stimulus guides the selected "level" in the compound frequency probe even if the prime and the probe as well as the tasks are completely different. It is important to note that in the current study, the same broadband Navon displays and the same compound grating probes were presented in the global and local attention blocks. The only difference across the blocks was the attended level while processing the prime and the attended SF while processing the probe. Another way to look at these effects is with respect to task switching. Ample evidence demonstrates that when a task changes from trial $n$ to trial $n+1$, there is a cost in performance relative to when the task does not change (Monsell, 2003). From this perspective, we can strengthen our interpretation by assuming that the level of processing (global-local) and the SF selected (LSF-HSF) share similar resources. Within this framework, allocating attention, for example, to the global level in trial $n$ and to the HSF in trial $n+1$ requires a more pronounced switch than allocating attention to the global level in trial $n$ and to the LSF in trial $n+1$.

The present pattern of results is also consistent with the notion of flexible scale usage in face and scene processing posited by Schyns and colleagues (Gosselin \& Schyns, 2001; Morrison \& Schyns, 2001; Oliva \& Schyns, 1997; Schyns \& Oliva, 1999). According to this view, the visual system does not necessarily operate in a fixed processing order (from LSF to HSF) on incoming information, but instead can flexibly use its effective spatial scales in an order determined by the diagnostic features relevant to the current task. Our results strongly endorse this view by demonstrating that the attended level in Navon patterns determined subsequent SF selectivity.

Finally, although hemispheric asymmetry was not examined in the current study, much of the research on processing hierarchical displays was derived from studies of hemispheric laterality and, indeed, the present findings bear indirect relevance to that literature. Specifically, the current data are consistent with theories, like the double filtering by frequency (DFF) theory (Ivry \& Robertson, 1998), proposing that hemispheric asymmetry in global versus local processing is a consequence of the asymmetrical filtering of relatively lower versus relatively higher frequency information. Central to DFF theory is the notion that the two cerebral hemispheres do not have an asymmetrical bias for the perceptual processing of SFs per se, but that they asymmetrically filter relatively LSF versus relatively HSF information after attention selects the task-relevant frequency range. The fact that we found an interaction between attention to hierarchical level and SF selectivity using identical compound gratings that varied peripheral and central presentation across experiments is consistent with this idea. However, independent of the DFF theory, the present data clearly demonstrate a robust relationship between the attended level in a multilevel object and the order of selection of SFs in an image. 


\section{Acknowledgments}

This work was supported by National Institute of Mental Health Grant Ro1 MH 64458 to Lynn C. Robertson and Shlomo Bentin. We thank Allison Yamanashi for help with running participants.

\section{References}

Delis DC, Robertson LC, Efron R. Hemispheric specialization of memory for visual hierarchical stimuli. Neuropsychologia 1986;24:205-214. [PubMed: 3714025]

De Valois, RL.; De Valois, K. Spatial vision. New York: Oxford University Press; 1988.

Fink GR, Halligan PW, Marshall JC, Frith CD, Frackowiak RS, Dolan RJ. Neural mechanisms involved in the processing of global and local aspects of hierarchically organized visual stimuli. Brain 1997;120(Pt. 10):1779-1791. [PubMed: 9365370]

Gosselin F, Schyns P. Bubbles: A technique to reveal the use of information in recognition tasks. Vision Research 2001;41:2261-2271. [PubMed: 11448718]

Han S, Chen L. Processing of global and local properties-An analysis with event-related brain potentials. Science in China, Series C, Life Sciences 1996;39:179-188.

Han S, Liu W, Yund EW, Woods DL. Interactions between spatial attention and global-local feature selection: An ERP study. NeuroReport 2000;11:2753-2758. [PubMed: 10976957]

Han S, Weaver JA, Murray SO, Kang X, Yund EW, Woods DL. Hemispheric asymmetry in globallocal processing: Effects of stimulus position and spatial frequency. NeuroImage 2002;17:12901299. [PubMed: 12414268]

Heinze HJ, Hinrichs H, Scholz M, Burchert W, Mangun GR. Neural mechanisms of global and local processing: A combined PET and ERP study. Journal of Cognitive Neuroscience 1998;10:485-498. [PubMed: 9712678]

Heinze HJ, Munte TF. Electrophysiological correlates of hierarchical stimulus processing: Dissociation between onset and later stages of global and local target. Neuropsychologia 1993;31:841-852. [PubMed: 8413904]

Henriksson L, Nurminen L, Hyva“rinen A, Vanni S. Spatial frequency tuning in human retinotopic visual areas. Journal of Vision 2008;8(10):1-13. [PubMed: 19146347]

Ivry, RB.; Robertson, LC. The two sides of perception. Cambridge, MA: MIT Press; 1998.

Lamb MR, Robertson LC, Knight RT. Attention and interference in the processing of global and local information: Effects of unilateral temporal-parietal junction lesions. Neuropsychologia 1989;27:471-483. [PubMed: 2733820]

Lamb MR, Yund EW, Pond HM. Is attentional selection to different levels of hierarchical structure based on spatial frequency? Journal of Experimental Psychology: General 1999;128:88-94. [PubMed: 10100393]

Martinez A, Moses P, Frank L, Buxton R, Wong E, Stiles J. Hemispheric asymmetries in global and local processing: Evidence from fMRI. NeuroReport 1997;8:1685-1689. [PubMed: 9189915]

Monsell S. Task switching. Trends in Cognitive Sciences 2003;7:134-140. [PubMed: 12639695]

Morrison DJ, Schyns PG. Usage of spatial scales for the categorization of faces, objects, and scenes. Psychonomic Bulletin \& Review 2001;8:434-469.

Navon D. Forest before trees: The precedence of global features in visual perception. Cognitive Psychology 1977;9:353-383.

Oliva A, Schyns PG. Coarse blobs or fine edges: Evidence that information diagnosticity changes the perception of complex visual stimuli. Cognitive Psychology 1997;63:72-107. [PubMed: 9325010]

Ostwald D, Lam JM, Li S, Kourtzi Z. Neural coding of global form in the human visual cortex. Journal of Neurophysiology 2008;99:2456-2469. [PubMed: 18322002]

Robertson LC. Attentional persistence for features of hierarchical patterns. Journal of Experimental Psychology: General 1996;125:227-249. [PubMed: 8751819]

Robertson LC. Spatial frequencies as a medium for guiding attention: Comment on Lamb, Yund, and Pond (1999). Journal of Experimental Psychology: General 1999;128:95-98. [PubMed: 10100394]

Robertson LC, Delis DC. "Part-whole" processing in unilateral brain-damaged patients: Dysfunction of hierarchical organization. Neuropsychologia 1986;24:363-370. [PubMed: 3736818] 
Robertson LC, Ivry R. Hemispheric asymmetries: Attention to visual and auditory primitives. Current Directions in Psychological Science 2000;9:59-63.

Robertson LC, Lamb MR. Neuropsychological contributions to theories of part/whole organization. Cognitive Psychology 1991;23:299-330. [PubMed: 2055002]

Robertson LC, Lamb MR, Knight RT. Effects of lesions of temporal-parietal junction on perceptual and attentional processing in humans. Journal of Neuroscience 1988;8:3757-3769. [PubMed: 3193178]

Schyns PG, Oliva A. Dr. Angry and Mr. Smile: When categorization flexibly modifies the perception of faces in rapid visual presentation. Cognition 1999;69:243-265. [PubMed: 10193048]

Sergent J. The cerebral balance of power: Confrontation or cooperation? Journal of Experimental Psychology: Human Perception and Performance 1982;8:253-272. [PubMed: 6461721]

Shulman GL, Sullivan MA, Gish K, Sakoda WJ. The role of spatial-frequency channels in the perception of local and global structure. Perception 1986;15:259-273. [PubMed: 3797200]

Shulman GL, Wilson J. SF and selective attention to local and global information. Perception 1987;16:89-101. [PubMed: 3671045]

Townsend, JT.; Ashby, FG. Stochastic modelling of elementary psychological processes. New York: Cambridge University Press; 1983.

Weissman DH, Woldorff MG. Hemispheric asymmetries for different components of global-local attention occur in distinct temporoparietal loci. Cerebral Cortex 2005;15:870-876. [PubMed: 15459080] 




Figure 1.

Example trial. Participants performed two tasks in alternating sequence. First, two Navon figures appeared on each side of fixation until response (prime). Participants indicated whether they differed at the global or local level (in separate blocks of trials). Following the prime, two compound frequency gratings appeared until response (probe). Participants reported the orientation of the lower spatial frequency (LSF) or higher spatial frequency (HSF) grating (in separate blocks of trials). 




Figure 2.

Average reaction times (RTs) to low spatial frequency (LSF) and high spatial frequency (HSF) targets in compound grating probes during the global and local attention blocks in Experiment 1. 


\section{Experiment 1: Individual participant data $(N=16)$}

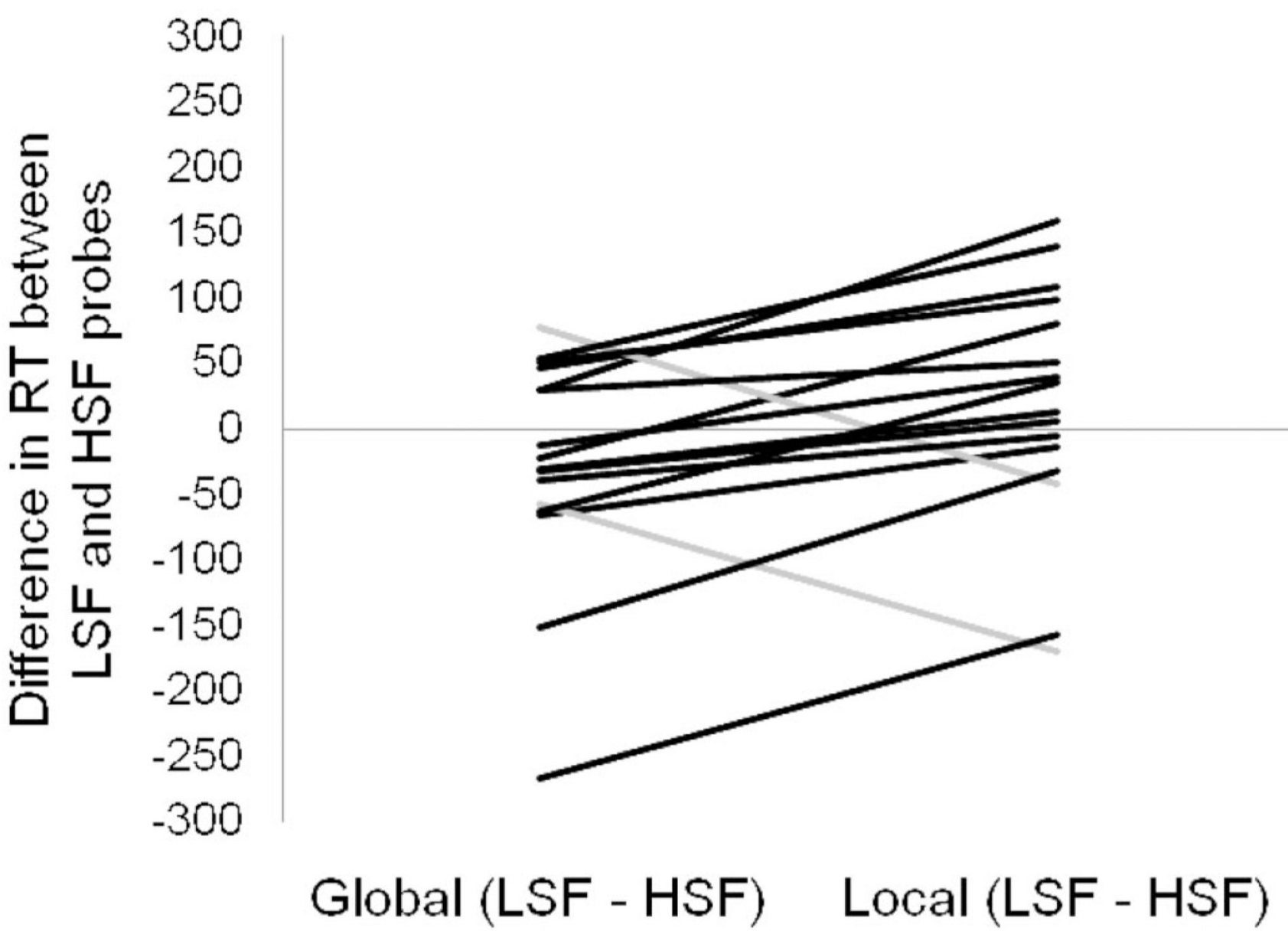

Figure 3.

Individual participant data from Experiment 1 showing the difference between reaction times (RTs) to low spatial frequency (LSF) targets minus high spatial frequency (HSF) targets in the global and local attention blocks. For all but two participants, this difference was greater in the local than global block, as evidenced by the increasing slope. The two participants who showed the opposite effect are shown in gray. 


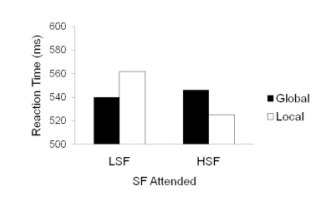

Figure 4.

Average reaction times (RTs) to low spatial frequency (LSF) and high spatial frequency (HSF) targets in compound grating probes during the global and local attention blocks in Experiment 2A. 


\section{Experiment 2: Individual participant data $(N=16)$}

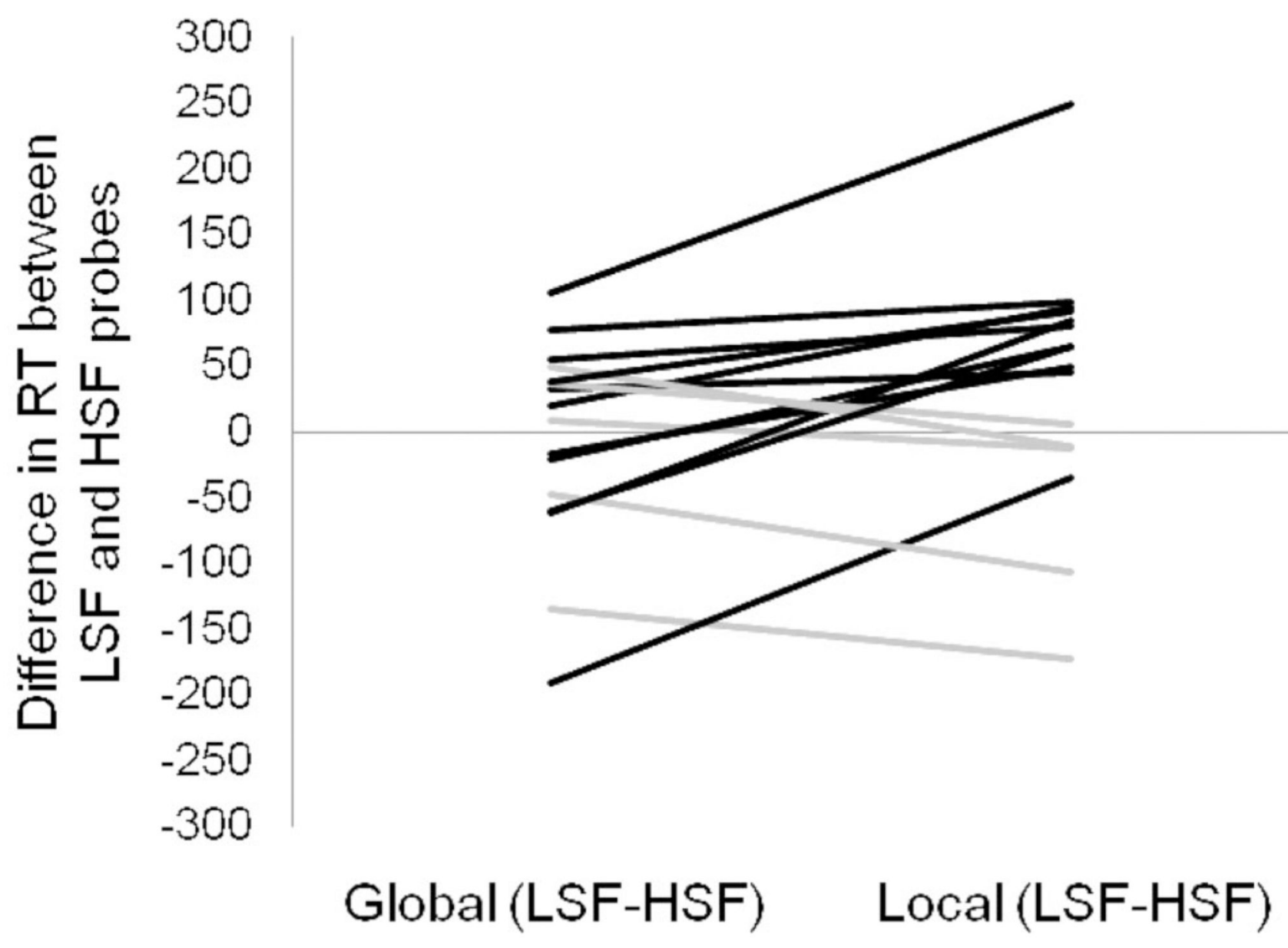

Figure 5.

Individual participant data from Experiment $2 \mathrm{~A}$ showing the difference between reaction times (RTs) to low spatial frequency (LSF) targets minus high spatial frequency (HSF) targets in the global and local attention blocks. For most participants (shown in black), there is an increasing slope, indicating an increased difference in the local versus the global block. Participants who did not reflect this pattern are shown in gray. 

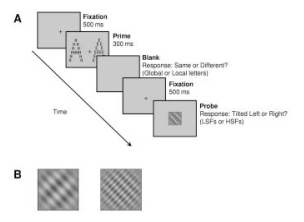

Figure 6.

Example trial and probe stimuli from Experiment 3. (A) Trial timing was identical to Experiment 2, as was the presentation of a single grating probe at fixation. (B) Examples of the absolute LOW (shown on the left) and absolute HIGH (shown on the right) compound grating probes. In both examples, the 1.8 cycle/degree grating is shown tilted to the left (not drawn to scale). It is the relatively higher SF of the set in the absolute LOW grating and the relatively lower SF of the set in the absolute HIGH grating. 


$$
1
$$

Figure 7.

Average reaction times (RTs) to relatively low spatial frequency (LSF) and relatively high spatial frequency (HSF) targets (collapsed over absolute spatial frequency) in compound grating probes during the global and local attention blocks in Experiment 3. 

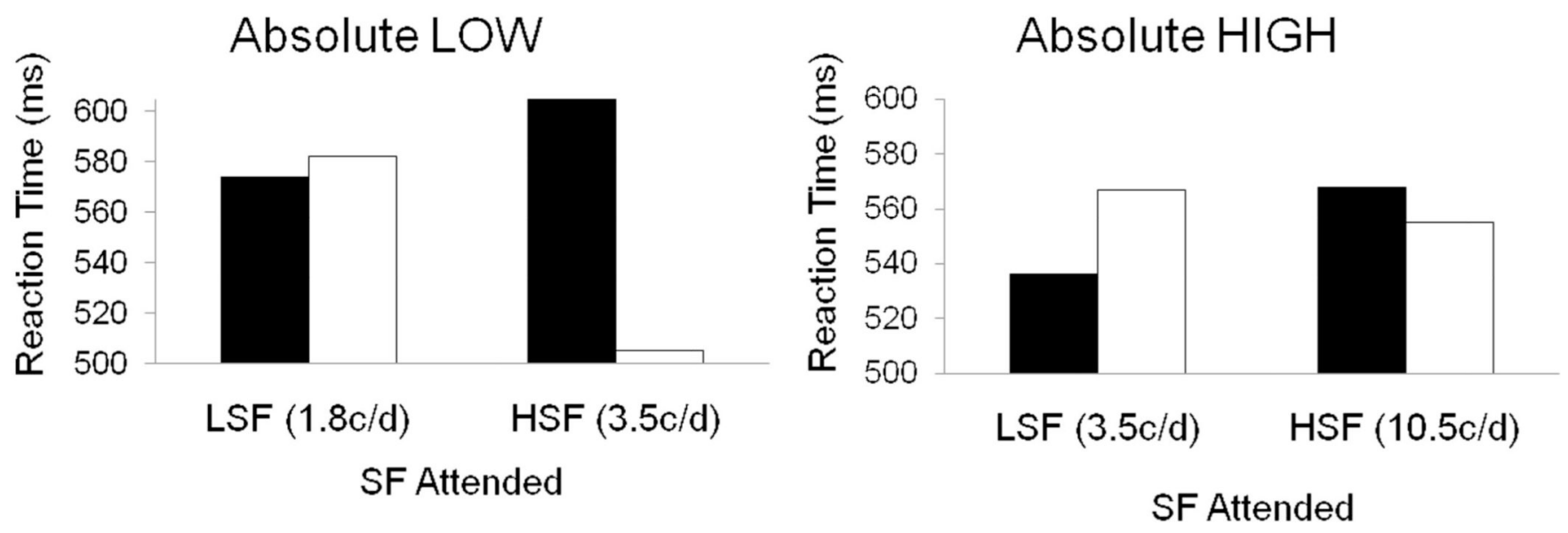

\section{Global}

Local

Figure 8.

Average reaction times (RTs) to relatively low spatial frequency (LSF) and relatively high spatial frequency (HSF) targets in compound grating probes during local and global attention blocks in Experiment 3, shown separately for absolute LOW and absolute HIGH grating conditions. 




Page 24 